\title{
Stark broadening of neutral calcium spectral lines
}

\author{
M.S. Dimitrijević ${ }^{1}$ and S. Sahal-Bréchot ${ }^{2}$ \\ 1 Astronomical Observatory, Volgina 7, 11000 Belgrade, Serbia, Yugoslavia \\ ${ }^{2}$ Laboratoire "Astrophysique, Atomes et Molécules", Département Atomes et Molécules en Astrophysique, \\ Unité associée au CNRS 812, Observatoire de Paris-Meudon, 92190 Meudon, France
}

Received June 24; accepted September 6, 1999

\begin{abstract}
Using the semiclassical perturbation approach, we have calculated electron-, proton-, He II-, Mg II-, Si II- and Fe II-impact line widths and shifts for $189 \mathrm{Ca}$ I multiplets as a function of temperature and perturber density. Perturbers selected here are the main perturbers in solar atmospheres. Obtained results have been compared with the existing theoretical and experimental data.
\end{abstract}

Key words: atomic data - line: profiles

\section{Introduction}

Calcium lines are present in solar (see e.g. Moore et al. 1966; Holweger 1972; Grevesse 1984) and stellar spectra (see e.g. Merrill 1956; Adelman \& Davis Philip 1992). Adelman \& Davis Philip (1992) have found, for example, neutral calcium lines in the Gamma Geminorum spectrum and Trimble (1991) reports the presence of this product of alpha processes in SN 1987 A ejecta. Consequently, Stark broadening data of neutral calcium spectral lines are of interest for stellar plasma as well as for laboratory plasma research and modeling. Neutral calcium spectral lines broadened by Stark effect have been investigated experimentally (Kusch \& Pritschow 1970; Hühn \& Kusch 1973) and theoretically (Griem 1974; Marassinghe \& Lovett 1986). Stark-effect interaction constants for seven solar calcium lines, whose abundances are sensitive to Stark broadening, have been estimated by Holweger (1972).

In accordance with our project (see e.g. Dimitrijević 1996a) to make available to astrophysicists and physicists an as large as possible set of reliable semiclassical Stark

Send offprint requests to: M.S. Dimitrijević

e-mail: mdimitrijevic@aob.bg.ac.yu

* Tables 1-3 are only available in electronic form: The material published electronically can be accessed: by $\mathrm{ftp}$ at cdsarc.u-strasbg.fr (130.79.128.5) or via http://cdsweb.ustrasbg.fr/Abstract.html broadening data needed for the investigation, diagnostics and modeling of various plasmas in astrophysics, physics and technology, we have calculated within the semiclassical-perturbation formalism (Sahal-Bréchot 1969a,b see also Sahal-Bréchot 1974; Dimitrijević \& Sahal-Bréchot 1984) electron-, proton-, ionized helium-, ionized magnesium-, ionized silicon-, and ionized ironimpact line widths and shifts for 189 neutral calcium multiplets. Consequently, the relevant data for all principal perturbers in the solar plasma are provided.

\section{Results and discussion}

For the determination of Stark broadening parameters (the full line width at half maximum - $W$ and the line shift $-d$ ) of neutral calcium, the semiclassical perturbation formalism has been used. This formalism, as well as the corresponding computer code (Sahal-Bréchot 1969a,b), have been updated and optimized several times (Sahal-Bréchot 1974; Fleurier et al. 1977; Dimitrijević \& Sahal-Bréchot 1984; Dimitrijević et al. 1991; Dimitrijević \& Sahal-Bréchot 1996). The calculation procedure, with the discussion of updatings and validity criteria, has been briefly reviewed in Dimitrijević (1996, 1997). Atomic energy levels needed for calculations have been taken from Sugar \& Corliss (1979). The oscillator strengths have been calculated within the Coulomb approximation (Bates \& Damgaard 1949, and the tables of Oertel \& Shomo 1968). For higher levels, the method of Van Regemorter et al. (1979) has been used.

All results are for temperatures from 2500 up to 50000 K. In Table 1 (accessible only in electronic form), electron-, proton-, and He II-impact broadening parameters for $189 \mathrm{Ca}$ I multiplets for perturber density of $10^{11} \mathrm{~cm}^{-3}$ are shown. Data for electron-, proton-, and He II-impact broadening parameters for Ca I for perturber densities of $10^{12} \mathrm{~cm}^{-3}-10^{19} \mathrm{~cm}^{-3}$ are shown in Table 2 (accessible only in electronic form). Table 3 (accessible only in electronic form) shows Mg II-, Si II-, and 
Fe II-impact broadening parameters for 189 Ca I multiplets, for perturber density of $10^{11} \mathrm{~cm}^{-3}$.

Stark broadening data for densities lower than for tabulated data, are proportional to the perturber density. Moreover, we present in Tables 1-3 as well, a parameter $C$ (Dimitrijević \& Sahal-Bréchot 1984), which gives an estimate for the maximum perturber density for which the line may be treated as isolated, when it is divided by the corresponding full width at half maximum. The validity of the impact approximation has been estimated for data shown in Tables 1-3, by checking if the collision volume $(V)$ multiplied by the perturber density $(N)$ is much less than one (Sahal-Bréchot 1969a,b). Values for $N V>0.5$ are not given and values for $0.1<N V \leq 0.5$ are denoted by an asterisk. When the impact approximation is not valid, the ion broadening contribution may be estimated by using the quasistatic approach (Sahal-Bréchot 1991 or Griem 1974). In the region between where neither of these two approximations is valid, a unified type theory should be used. For example in Barnard et al. (1974), a simple analytical formula for such a case is given. The accuracy of the results obtained decreases when broadening by ion interactions becomes important.

There are two experimental studies reporting results of measurements of neutral calcium line Stark broadening parameters (Kusch \& Pritschow 1970; Hühn \& Kusch 1973). Kusch \& Pritschow (1970) have investigated experimentaly Stark widths and shifts of four neutral calcium lines from plasma produced in a pulsed capillary discharge, and reported results for an electron density of $10^{18} \mathrm{~cm}^{-3}$. Only for one line (the $5188.8 \AA$ line from the $4 \mathrm{p}^{1} \mathrm{P}^{\circ}-5 \mathrm{~d}^{1} \mathrm{D}$ multiplet) exist a sufficiently complete set of atomic data needed for an adequate application of our approach. For this line, however, the impact approximation condition is not satisfied for the reported electron density. Hühn \& Kusch (1973) published experimental Stark widths for the $4318.6 \AA$ and $4425.4 \AA$ lines from the $4 \mathrm{~s} 4 \mathrm{p}^{3} \mathrm{P}^{\circ}-4 \mathrm{p}^{2}{ }^{3} \mathrm{P}$ and $4 \mathrm{~s} 4 \mathrm{p}^{3} \mathrm{P}^{\circ}-4 \mathrm{~s} 4 \mathrm{~d}^{3} \mathrm{D}$ multiplets, from plasma produced in a high pressure arc. Only the $4425.4 \AA$ line may be compared with our results. For an electron density of $10^{17} \mathrm{~cm}^{-3}$ and the temperature $10000 \mathrm{~K}$, Hühn \& Kusch (1973) obtain for the full width at half maximum the value of $0.29 \AA$, while our result is $1.26 \AA$. The semiclassical result of Griem (1974) is $1.51 \AA$. As one can see, the agreement between the experimental result and both theories is very poor. The differences between the calculation methods used by us and those of Griem (1974), have been discussed in detail in Dimitrijević \& Sahal-Bréchot (1996). The reasons for such large difference between experiment and theory have been discussed in detail in Konjević \& Roberts (1976), and the principal point of their remarks on the experiment is the lack of an independent measurement of electron density.

It is surprising that in spite of the importance of neutral calcium lines, there are not more experimental data. Reliable experimental data for Stark broadening of Ca I lines will be important for the check of theory, as well as for abundance determinations and other problems in astrophysics and plasma physics.

Acknowledgements. This work has been supported by the Ministry of Science and Technology of Serbia through the project "Astrometrical, Astrodynamical and Astrophysical Researches".

\section{References}

Adelman S.J., Davis Philip A.G., 1992, PASP 104, 316

Barnard A.J., Cooper J., Smith E.W., 1974, J. Quant. Spectrosc. Radiat. Transfer 14, 1025

Bates D.R., Damgaard A., 1949, Trans. Roy. Soc. London, Ser. A 242, 101

Dimitrijević M.S., 1996, Zh. Prikl. Spektrosk. 63, 810

Dimitrijević M.S., 1997, Astrophys. Space Sci. 252, 415

Dimitrijević M.S., Sahal-Bréchot S., 1984, J. Quant. Spectrosc. Radiat. Transfer 31, 301

Dimitrijević M.S., Sahal-Bréchot S., 1996, Phys. Scr. 54, 50

Dimitrijević M.S., Sahal-Bréchot S., Bommier V., 1991, A\&AS 89, 581

Fleurier C., Sahal-Bréchot S., Chapelle J., 1977, J. Quant. Spectrosc. Radiat. Transfer 17, 595

Grevesse N., 1984, Phys. Scr. T8, 49

Griem H.R., 1974, Spectral Line Broadening by Plasmas. Academic Press, New York

Holweger H., 1972, Solar Phys. 25, 14

Hühn R., Kusch H.J., 1973, A\&A 28, 159

Konjević N., Roberts J.R., 1976, J. Phys. Chem. Ref. Data 5, 209

Kusch H.J., Pritschow H.P., 1970, A\&A 4, 31

Merrill P.W., 1956, Lines of the Chemical Elements in Astronomical Spectra, Carnegie Inst. of Washington. Publication 610, Washington DC

Moore C.E., Minnaert M.G.J., Houtgast J., 1966, The Solar Spectrum $2935 \AA$ to $8770 \AA$, NBS Monographs 61, U.S. Department of Commerce, NBS, Washington DC

Oertel G.K., Shomo L.P., 1968, ApJS 16, 175

Sahal-Bréchot S., 1969a, A\&A 1, 91

Sahal-Bréchot S., 1969b, A\&A 2, 322

Sahal-Bréchot S., 1974, A\&A 35, 321

Sahal-Bréchot S., 1991, A\&A 245, 322

Sugar J., Corlis C., 1979, J. Phys. Chem. Ref. Data 8, 865

Trimble V., 1991, The Origin and Abundance of the Chemical Elements Revisited, Astron. Astrophys. Review, No. 3, 1

Van Regemorter H., Hoang Binh Dy, Prud'homme M., 1979, J. Phys. B 12, 1073 